\title{
Solar latitudinal distortions: From observations to theory
}

\author{
S. Lefebvre and J. P. Rozelot
}

\author{
Observatoire de la Côte d'Azur (OCA), GEMINI, Avenue Copernic, 06130 Grasse, France \\ e-mail: sandrine.lefebvre@obs-azur.fr
}

Received 29 July 2003 / Accepted 18 December 2003

\begin{abstract}
Solar diameters have been measured from different ground-based instruments on different sites all around the world. There are values dating back to three centuries ago, but the revival of interest began in the 1970s when it was claimed that a temporal periodic modulation had been found. The interest of such measurements, pinpointed from only two decades, may not lie in these temporal variations, but in the fact that a latitudinal heliographic dependence may exist. Such a solar shape distortion has been deduced from the analysis of solar astrolabe data sorted by heliographic latitudes, but observational evidence has also been obtained by means of a scanning heliometer (Pic du Midi Observatory). Latitudinal dependence implies sub-surfacic physical mechanisms and can be explained theoretically. Thus, in spite of the fact that ground-based observations are altered by seeing effects that may amplify or superimpose noise, it can be advanced that the solar shape is not a pure spheroid. We present here a new theory based upon the thermal-wind equation, which explains the observed distorted solar shape. Using the $W$ parameter (called here asphericity-luminosity parameter), we show that large negative values ( $W$ ranging from around -0.075 up to -0.6 ) leading to a prolate Sun, are unlikely. The best range of $W$ lies between around -0.075 and +0.6 . Concerning observations, only space missions (or balloon flights) will be able to reach a clear conclusion. A space mission called PICARD is scheduled to be launched by 2008: one of its major aims is to measure these asphericities with astrometric precision.
\end{abstract}

Key words. Sun: general - Sun: fundamental parameters - Sun: solar wind

\section{Introduction}

Since a few years, the idea that the outer visible shape of the Sun is not a perfect ellipsoid seems to be more and more convincing. From an observational point of view, measurements of high precision, recently made at the Pic du Midi Observatory and during exceptional meteorological conditions, have suggested a distorted outer shape (Rozelot et al. 2003a,b; Rozelot \& Lefebvre 2003). Theoretically, the free surface is related to thermodynamic effects growing from the tachocline to the near surface. If all physical mechanisms occurring in the sub-surfacic layers were perfectly uniform (velocity rate, mass distribution, magnetic field...) the surface boundary of the Sun would be a perfect spheroid solely owing to (uniform) rotation. This is not the case, and a distorted shape is expected. Using a shallow-water model, Dikpati \& Gilman (2002) reached the same conclusion: a deformed outer sphere represents the "freetop" boundary of the solar fluid. In the light of these theoretical facts, we wondered if it might not be opportune to reconsider ground-based measurements, and instead of trying to search for a consistency in individual data acquired on different sites and at different epochs, to look at what could be reliable and directly comparable.

Another intriguing point is the value of the so-called octopole term $(l=4)$ for which the observations, either from

Send offprint requests to: J. P. Rozelot, e-mail: jean-pierre.rozelot@obs-azur.fr the ground or from space, do not match the theoretical value. For example, according to Lydon \& Sofia (1996), the observed $l=4$ term is greater than the theoretical one by a factor that may reach 4 orders of magnitude. As, in any theory of figure of rotating bodies, the outer shape is the combination of the successive gravitational moments, for example at first order $l=2$, then $l=4$, the result is that any increased value of the $l=4$ term allows the upper boundary of the Sun to vary with latitude. In the following, we will adopt the term "asphericities" to describe any departures from perfect sphericity of the Sun, and the outer visible shape of the Sun will be named "helioid".

In the second part of this paper we will briefly recall existing ground-based observations, and we will discuss them considering only their common properties, with respect to solar latitudinal variations. In the third section, we will propose a new theory based upon the thermal wind equation, which might explain the observed distortions. In the Sect. 4, we will be able to propose bounds on such asphericities and we will conclude by some perspectives to obtain observations in space to decide the issue.

\section{Solar radius ground-based observations}

There are many papers describing ground-based observations of the solar radius, mainly using the so-called modified Danjon astrolabe. These solar astrolabes operate now in France (Laclare et al. 1996), Chile (Noël 2002, 2003a), Brazil 
(Reis Neto et al. 2003) and Turkey (Gölbaşi et al. 2001). These measurements have been already analyzed and discussed, for instance by Ribes et al. (1991), Laclare et al. (1996), Noël (2002), Jilinski et al. (1999), Wittmann (2001), Emilio et al. (2000) and Pap et al. (2001) ${ }^{1}$. When data from different sources are compared over a fairly long period of time, they show strong disparities, both in amplitude and in phase. The reason, or reasons for the discrepancies have not yet been fully explained. They might due either to technical points (variable angle prism, CCD...), or to the atmospheric turbulence hiding faint solar effects. It is also true that, as the amount of data increases with time, it is possible to apply to these series increasing performant data analysis techniques. The results are sometimes impressive, leading for example to accuracies of a few milliarcseconds (mas) in data obtained near the sea level. The results, although frequently obtained on a patrol way, are not always readily available (except in one case); the reason usually advanced being the waiting for comparison with other solar indices. A critical look afterwards at all the solar astrolabe data remains to be taken. However, the question deserves to be tackled, with caution: the results so far obtained from the ground may conceal interesting features. On the other hand, astrometric space data are not yet available and it is clear that data which will be obtained from spacecraft will make it possible to give a definite answer to the question. We will return to this point in Sect. 5.

Serious analyses have been made that render the question of the variability of the solar radius with time a question of astrophysical interest. Let us mention the solar variability model presented by Sofia \& Li (2001), which permits the reconstruction of solar irradiance, and which is partially based on longterm variations of the solar radius. One may also ask what would be an upper bound of the radius variations, for which, above this limit, one will be sure that an astrophysical phenomenon could no more be observed. In the past, some authors (such as Beardsley \& Hill 1987) have searched in that direction, the advance of the perihelion of Mercury being taken as a test. Unfortunately, at that time, this test was not sensitive enough due to the size of the errors (in the observed values of the perihelion advances of planets). A third example can be found in Rozelot \& Bois (1998): they showed that the indirect signature of the solar quadrupole moment on the Moon's rotational motion leads to an upper limit of this gravitational $l=2$ moment, which in turn limits the variations of the solar radius to some 50 mas, a value certainly still high, but indicating that variations must exist, otherwise there would be lunar librations of so high amplitude that they could never be observed.

It seems to us that a direct comparison of solar astrolabe data, even through sophisticated filtering techniques, do not solve the problem of their inconstancy. Taking into account recent progress concerning physical mechanisms inside the Sun, which lead to a distorted visual shape of the Sun, we will attempt to tackle the subject in another way.

Firstly, note that solar astrolabe observers do not measure the same diameter at the same time. This is due to the latitude

\footnotetext{
1 This list is not exhaustive; number other references can be found in the above quoted papers.
}

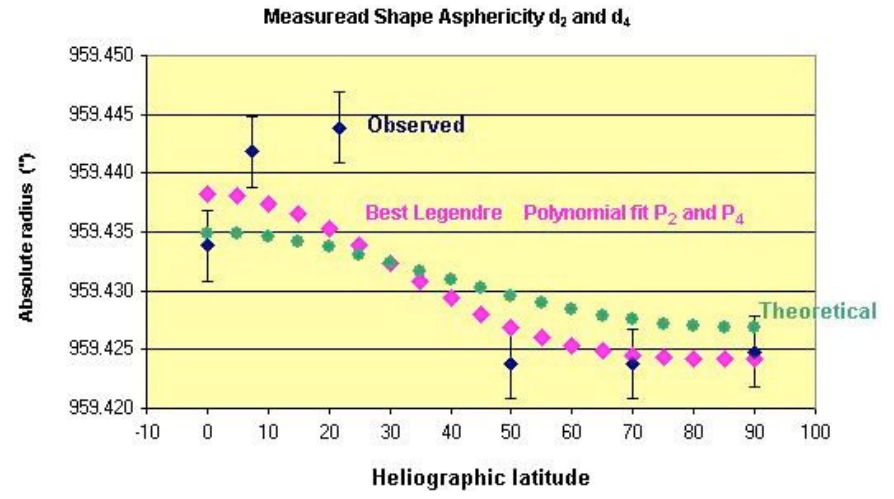

Fig. 1. The solar limb shape variations as observed at the Pic du Midi Observatory from September 3rd to 6th, 2001. The amplitude of the variations does not exceed 24 mas between the highest and the lowest point of the error bars. The overall shape of the Sun remains oblate but a bulge is observed near the royal zones $\left(30^{\circ}-40^{\circ}\right.$, a zone where spots appear at the beginning of the solar cycle) followed by a depression. After Rozelot et al. (2003b).

of the observing site and to the fact that diameters are locally measured through the vertical. Thus, to compare data series, one must first group them into heliographic latitude blocks. Similarities are then clearly found. An example can be found in Noël (2003b), where a distorted shape of the limb appears anticorrelated with the surface effective temperature as measured by Kuhn (1988). Other techniques lead to the same conclusions. As mentioned in the introduction, departures from sphericity were recently observed from the ground by means of the scanning heliometer of the Pic du Midi Observatory (Rösch et al. 1996) and are reported in Fig. 1 (from Rozelot et al. 2003a or 2003b).

Inspection of the two curves given in Figs. 1 and 2 deduced from ground-based measurements shows overall similarity with a few discrepancies, which can be due to the measuring difficulties. The main feature is a departure from a perfect sphere; the asphericities can be described as follows. A bulge is visible extending from the equator up to the mid latitudes, around $45^{\circ}$. This bulge is followed by a depressed zone at higher latitudes, from $60^{\circ}$ to $80^{\circ}$. The overall shape remains oblate. Note that departures from a sphere were also suspected from solar astrolabe measurements made at Calern observatory (France) as early as 1996 (Laclare et al. 1996), and at Santiago (Chile) by Noël (1999, 2002).

We are indeed conscious of the difficulties in measuring such asphericities, and of the considerable errors associated with measurements from different sites. The main source of error is the atmospheric turbulence and the true difficulty is to distinguish between the variations due to the atmosphere and those of true solar origin. A more complete study of the atmospheric effects on solar diameter measurements has been made by Irbah et al. (2003) who show that the observation conditions have more impact on the errors than on the measurements of the diameter mean value. Even if the data are certainly affected by the turbulence effects, it would be unlikely that rather similar results found by different observers on different sites (and using different techniques) would not reflect a real solar effect. 


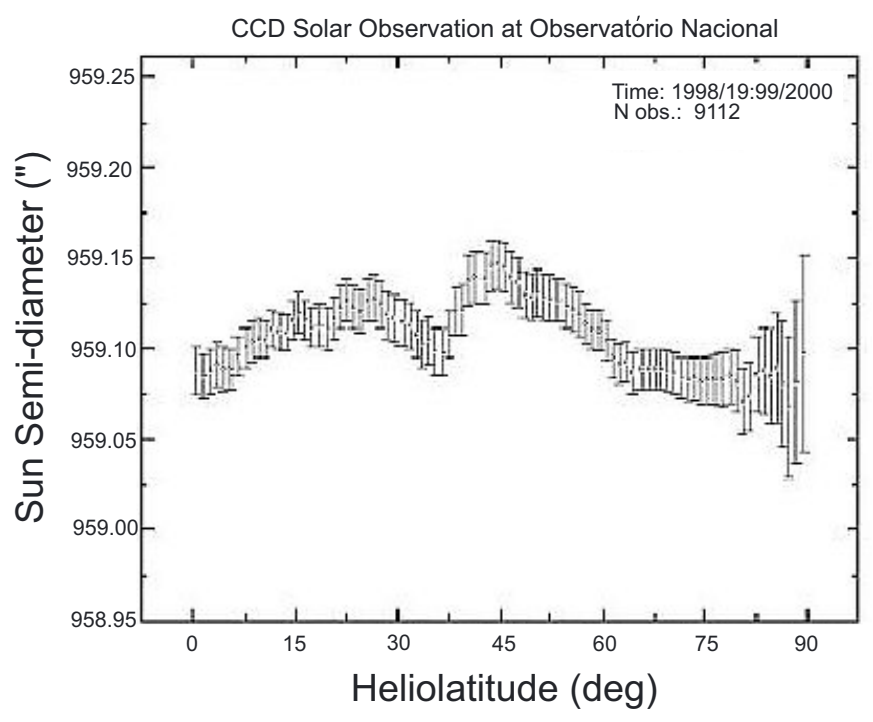

Fig. 2. Solar limb shape variations as observed from the ground by the solar astrolabe. The measurements were made by Reis Neto et al. (2003) in Rio de Janeiro (Brazil). A bulge can be seen extending from the equator to the end of the royal zones (around $45^{\circ}$ ) followed by a depression between $60^{\circ}-80^{\circ}$ (by courtesy of Andrei, personal communication, 2002; see also Reis Neto et al. 2003).

Moreover, recent theoretical progress concerning the functioning of the tachocline has shown that the instability of the differential rotation as a function of the effective gravity of the successive internal stratified shells leads to latitudinal variations of the outer shape of the Sun (Dikpati \& Gilman 2001, 2002; Lefebvre \& Rozelot 2003).

Such distortions have not yet been observed from space with any instrument (SOHO/MDI or SDS balloon measurements). However, we should recall that SOHO/MDI has a resolution of about 2 arcsec per pixel, which is probably too low to show such distortions (which were not searched for).

In summary, we conclude that a comparison of all data from several techniques and from different sites indicates that a bulge is visible on the solar surface around the royal zones (around $35^{\circ}-45^{\circ}$, a zone where spots appear at the beginning of the solar cycle), with a depressed zone at higher latitudes. The difference between the equatorial and polar radius remains positive. The helioid thus has a complex shape, where departures from the sphere probably do not exceed twenty mas, which must now be explained.

\section{A new theoretical approach to pinpoint departures to sphericity}

\subsection{Preamble}

Consider a single star that rotates about a fixed direction in space, with some assigned angular velocity; the resulting shape is an oblate figure. However, for the Sun, a slowly rotating star, with differential rotation on the surface and at depth, the following questions arise: what is the geometrical shape of the free boundary and what is the form of the surfaces upon which the physical variables (such as pressure, density, ...) remain constant? How do these equilibrium levels depend on the angular velocity distribution?

\subsection{The thermal-wind relation}

For a better understanding of the question, let us first recall the the thermal-wind equation, which can also be found also in Gough \& McIntyre (1998) and Tassoul (2000).

Let $\boldsymbol{v}$ denote the velocity of the fluid in an inertial frame of reference, $\boldsymbol{g}$ the acceleration due to gravity, $\rho$ the density, $p$ the pressure, $\boldsymbol{f}$ the viscous force per unit volume and $V$ the gravitational potential; we start with the Navier-Stokes equation

$\frac{D \boldsymbol{v}}{D t}=\boldsymbol{g}-\frac{1}{\rho} \boldsymbol{g r a d}(p)+\frac{1}{\rho} \boldsymbol{f}(\boldsymbol{v})$,

which reduces to

$\frac{D v}{D t}+\operatorname{grad}(V)+\frac{1}{\rho} \operatorname{grad}(p)=\mathbf{0}$

under the following hypothesis:

- the Sun is an axisymmetric star, which rotates about a fixed direction in space with some assigned angular velocity;

- the motion is steady in time;

- molecular viscosity is negligible for large-scale motion; in this case, viscous transport (turbulence) can also be neglected.

Introducing the classical notations $(r, \theta, \phi)$ with respect to the rotation axis, in a frame rotating with angular velocity $\Omega$, and cylindrical polar coordinates $(s, z, \phi)$, where $z=r \cos (\theta)$ and $s=r \sin (\theta)$, we may write $\left(\boldsymbol{e}_{s}, \boldsymbol{e}_{z}, \boldsymbol{e}_{\phi}\right)$ for the rotating frame in cylindrical coordinates. Thus, the velocity $v$ has the form

$\boldsymbol{v}=\Omega s \boldsymbol{e}_{\phi}$,

and the hypothesis of constancy in time implies that

$\frac{D v}{D t}=-\Omega^{2} s e_{s}$

From Eq. (4), Eq. (2) becomes

$\operatorname{grad}(V)+\frac{1}{\rho} \operatorname{grad}(p)-\Omega^{2} \boldsymbol{s e}_{s}=\mathbf{0}$.

Taking the curl of Eq. (5), we obtain

$\operatorname{grad}\left(\frac{1}{\rho}\right) \wedge \operatorname{grad}(p)=\operatorname{grad}\left(\Omega^{2} s\right) \wedge \boldsymbol{e}_{s}$

This last equation is the thermal wind relation, which relates the $z$ dependence of the angular velocity $\Omega$ to the baroclinicity of the system. Developing Eq. (6) by choosing for convenience cylindrical coordinates for the left member, as introduced by Gough \& McIntyre (1998), we obtain

$-\frac{1}{r \rho^{2}}\left(\frac{\partial \rho}{\partial r} \frac{\partial p}{\partial \theta}\right)=2 \Omega s \frac{\partial \Omega}{\partial z}$

by assuming that the density $\rho$ is independent of the colatitude $\theta$. 
Table 1. Empirical rotation coefficients $\Omega_{\mathrm{eq}}, \Omega_{2}$ and $\Omega_{4}$ (in $\mu \mathrm{rad} / \mathrm{s}$ ) according to Allen (2000) and some other authors. These coefficients have been used for the computation of our model shown in Figs. 3 and 4.

\begin{tabular}{|c|c|c|c|c|}
\hline References & Method & $\Omega_{\mathrm{eq}}$ & $\Omega_{2}$ & $\Omega_{4}$ \\
\hline \multicolumn{5}{|c|}{ From tracers } \\
\hline$\left\{\begin{array}{l}\text { Duvall (1980) } \\
\text { Snodgrass et al. (1990) }\end{array}\right.$ & Supergranular pattern & 2.972 & -0.483 & -0.360 \\
\hline Komm et al. (1993) & Small magnetic features & 2.913 & -0.404 & -0.422 \\
\hline Kosovichev (1996) & Helioseismology & 2.843 & -0.382 & -0.461 \\
\hline Kuiper (1972) & Sunspots & 2.913 & -0.283 & -0.269 \\
\hline Makarov et al. (1997) & $\mathrm{H} \alpha$ line map & 2.824 & -0.190 & -0.067 \\
\hline Snodgrass (1983) & Magnetic field pattern & 2.903 & -0.483 & -0.327 \\
\hline Wilson et al. (1996) & Helioseismology & 2.903 & -0.484 & -0.264 \\
\hline Deng et al. (1999) & Polar magnetic elements & 2.828 & -0.453 & -0.360 \\
\hline \multicolumn{5}{|c|}{ From the Doppler effect in solar lines } \\
\hline Snodgrass et al. (1984) & Surface plasma & 2.850 & -0.343 & -0.475 \\
\hline
\end{tabular}

Equation (7) relates the balance of the pressure gradient and the Coriolis force. To express this relation in temperature instead of pressure, we assume hydrostatic equilibrium and a perfect gas law that yields the following thermal wind equation:

$\frac{g}{r T}\left(\frac{\partial T}{\partial \theta}\right)_{r}=2 \Omega s\left(\frac{\partial \Omega}{\partial z}\right)_{s}$.

To sum up, the balance is expressed by the zonal component of the steady axisymmetric inviscid vorticity equation, yielding an appropriately generalized "thermal-wind" (more aptly, "thermal-shear") equation. The thermal wind equation links the shear of angular velocity to the latitudinal variation of the temperature field $T$. Note that such latitudinal variations of $T$ have already been measured on the surface of the Sun (Kuhn 1985, 1988).

\subsection{Relation with the latitudinal variation of the radius}

At the surface of the Sun (namely in the photosphere), the rotation law $\Omega$ is often described by a 3-term expansion

$\Omega(\theta)=\Omega_{\mathrm{eq}}+\Omega_{2} \cos ^{2}(\theta)+\Omega_{4} \cos ^{4}(\theta)$,

where $\Omega_{\mathrm{eq}}$ is the equatorial rotation rate and $\Omega_{\mathrm{i}}$, empirical coefficients fitting the observational data (see Table 1). These coefficients are often limited in latitude, to about $60^{\circ}$, because effects such as geometrical distortion, make it very difficult to adequately measure the rotation rates at high latitudes. Schou et al. showed that an extrapolation of such an expansion to higher latitudes will lead to a discrepancy with helioseismic observations (which are generally reliable to at least $75^{\circ}$ ). On the other hand, Deng et al. carefully measured the surface rotation rate at high latitudes $\left(55^{\circ}-85^{\circ}\right.$; see coefficients in Table 1) and found them to be consistent with helioseismic results.

It is known from helioseismology that the surface differential rotation goes on deeper with a radial gradient of the rotation rate $\frac{\partial \Omega}{\partial r}$ that is not null, and negative in the near-surface layers of the convection zone. The order of magnitude of this outward gradient is $-5.7 \times 10^{-16} \mathrm{~m}^{-1} \mathrm{~s}^{-1}$ at the equator. Moreover,
Corbard \& Thompson (2002) have shown that in a shell of about $0.02 R_{\odot}$ thickness, the radial gradient, constant (but negative) from the equator up to latitude $50^{\circ}$, cancels around this latitude and reverses to higher latitudes (the values are then extrapolated to the surface). Basu et al. (1999) deduced a similar behaviour from their analysis of the splitting of high-degree $f$ modes, but finding a reversal of the radial gradient in the zone above $0.994 R_{\odot}$. It follows from this analysis that the radial gradient can be neglected for our purpose (at least in this subsurfacic layer called the leptocline, Godier \& Rozelot 2001), which leads to

$\frac{\partial \Omega}{\partial z}=-\frac{\sin (\theta)}{r} \frac{\partial \Omega}{\partial \theta}$

Developing $T$ as usual into the form $T(\theta)=T_{\mathrm{e}}+\tilde{T}(\theta)$, where $T_{\mathrm{e}}$ is the effective temperature at the surface of the Sun, we obtain

$\frac{g}{r T_{\mathrm{e}}} \frac{\partial \tilde{T}}{\partial \theta} \approx-2 \Omega \sin ^{2}(\theta) \frac{\partial \Omega}{\partial \theta}$.

Following Sofia \& Endal (1980), we introduce the so-called parameter $W$ which links the relative variations of the solar radius $R$ to the relative variations of the irradiance $I$ as

$W=\frac{\mathrm{d} R / R}{\mathrm{~d} I / I}$

Bearing in mind Stefan's law $L=\sigma T^{4}$, we get

$\frac{\mathrm{d} R}{R}=W \frac{\mathrm{d} I}{I}=W \frac{\mathrm{d} L}{L} \approx \frac{4 W}{T_{\mathrm{e}}} \frac{\partial \tilde{T}}{\partial \theta} \mathrm{d} \theta$.

Finally, substituting Eq. (13) into Eq. (11) and introducing $R(\theta)=R_{\mathrm{e}}+\tilde{R}(\theta)$, where $R_{\mathrm{e}}$ is the initial ellipsoid, we obtain

$\frac{\mathrm{d} \tilde{R}}{\mathrm{~d} \theta} \approx-\frac{8}{g} W R r \sin ^{2}(\theta) \Omega \frac{\partial \Omega}{\partial \theta}$

This last equation appropriately describes the evolution of the solar radius with colatitude $\theta$. Integration of Eq. (14) requires an initial step of $R$, which can be taken as an ellipsoid with an initial value of $\Delta R_{\mathrm{i}}=R_{\mathrm{eq}}-R_{\mathrm{pol}}$, starting with sphericity. Then, 


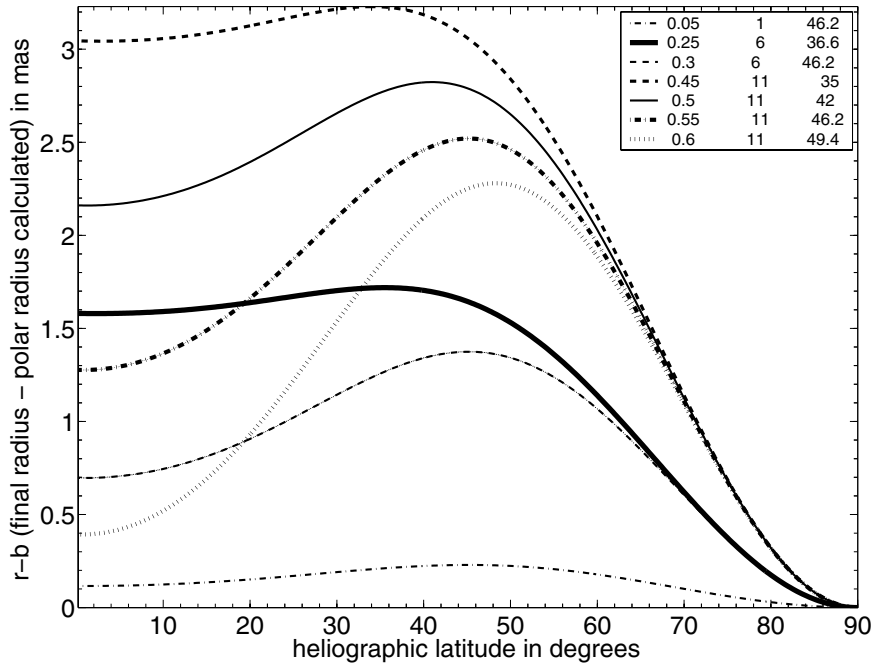

Fig. 3. Theoretical solar radius variations (with reference to the polar radius, in mas) versus heliographic latitude, in degrees. The successive columns in the legend indicate the value of $W$ and of the initial $\Delta R_{\mathrm{i}}$ (in mas) and, the latitude at which the maximum of each curve occurs, repectively. Note that the estimated maximum found for each curve is located between $30^{\circ}$ and $50^{\circ}$ latitude (the coefficients of the rotation law have been taken from Snodgrass 1983).

as both absolute values of $W$ and $\Delta R_{\mathrm{i}}$ have an upper bound, the integration can be limited to their most plausible values.

As we will see in the next section, values of $W$ has been taken ranging from -0.6 to +0.6 . We started with $\Delta R_{\mathrm{i}}=1$ mas, i.e. quasi sphericity, and we stopped with $\Delta R_{\mathrm{i}}=22$ mas, which can be considered as an upper initial value for the initial reference ellipsoid. $\Delta R_{\mathrm{i}}=7$ mas is what would be an initial ellipsoid if the Sun were rigidly rotating (without differential rotation) and $\Delta R_{\mathrm{i}}=10$ mas is one of the most plausible values. The two other values are intermediate steps of calculations.

\section{Results}

\subsection{Estimation of the "asphericity-luminosity parameter W"}

Today, both the absolute value and the sign of the "asphericityluminosity parameter $W$ " are still badly known. Different values are available in the literature, either theoretically calculated or measured from ground-based semi-diameter measurements, but they are not consistent. According to different authors, theoretical values range from $2 \times 10^{-4}$ (Spruit 1991), $5 \times 10^{-3}$ (Dearborn \& Blake 1980), $7.4 \times 10^{-2}$ (Sofia et al. 1979), to 0.2 or 0.5 if studies that locate mechanisms are at the base of the convective zone or in the core respectively (Gough 2001). From the observations the absolute value of $W$ varies from 0.85 (Rozelot 2001) to 0.2 (Picard web site).

To check our model described by Eq. (14), a first computation was made, using values of the coefficients of Eq. (9) taken from Snodgrass (1983); these values are close to those that Wilson et al. (1996) deduced from helioseismology. The results obtained for the latitudinal variations of the solar radius versus the heliographic latitude are shown in Fig. 3. The shape of the free surface clearly depends on the values of the pair $\left(W, \Delta R_{\mathrm{i}}\right)$. Note that the position of the maxima obtained is concentrated between $30^{\circ}$ and $50^{\circ}$ of heliographic latitude. The first curve $[0.05 ; 1]$, at the bottom of the diagram is unlikely, as the final difference obtained between the polar and equatorial radius is only of about 0.15 mas.

The two pairs ( $W=0.5$ and $\left.\Delta R_{\mathrm{i}}=11 \mathrm{mas}\right),(W=0.45$ and $\Delta R_{\mathrm{i}}=11$ mas) are the most likely values: the profiles of the outer layer are well marked, the latitudinal thickness occurring near the royal zones, at latitude $40^{\circ}$. The final difference between the polar and equatorial radius is about 2 to 3 mas, the outer global shape remaining oblate.

\subsection{Theoretical solar radius variations}

The model has also been tested to check if it is sensitive to the rotation law. Different values of the $\left(\Omega_{\mathrm{eq}}, \Omega_{2}, \Omega_{4}\right)$ triplet quoted in Table 1 have been used, deduced from Allen (2000) and some other authors. The results are given in the different graphs of Fig. 4. Except for the so-called "H $\alpha$-line map", all plots show a similar behaviour with a maximum occurring at the mid-latitudes. In the case of the " $\mathrm{H} \alpha$-line map", the height at which the line is formed is about $1000 \mathrm{~km}$ above the photosphere (see Fig. 1, Floyd 2003), that is to say far from the surface itself. In this case, it is not surprising to obtain a quasi ellipsoidal surface, for which the final $\Delta R$ is of about 7 mas (and for a $W$ value of about 0.5 ). The other graphs merely reflect the depth at which the differential rotation takes place. It has also been checked that results are not too much affected by using polar tracing elements that make it possible to deduce coefficients of Eq. (9) for high latitudes (for instance, $\theta_{\max }=41.4^{\circ}$ ) for $W=0.5$ and $\Delta R_{\mathrm{i}}=11$ mas; see below). In the following, we will continue to use the values of $\Omega_{\mathrm{i}}$ taken from Snodgrass (1983).

Figure 5 shows, plotted versus heliographic latitude, the boundary of the free surface obtained as a function of the initial $\Delta R_{\mathrm{i}}$. This figure indicates that, for values of $\Delta R_{\mathrm{i}}$ going from sphericity to an oblateness of 10 mas, large positive values of $W$ yield a final prolate Sun; such a scenario can certainly be rejected. By contrast, the two last figures (labelled 16 and 22 mas) show that negative values of $W$ increase the initial $\Delta R_{\mathrm{i}}$ to values that are certainly never observed. Our conclusion is that $\Delta R_{\mathrm{i}}$ must lie between 7 and 12 mas and $W$ is limited to a range of values between -0.075 and +0.6 . To explain the observed heliographic variations showing a maximum at around latitude $40^{\circ}$, the best pairs are $\left(\Delta R_{\mathrm{i}}=10\right.$ (Fig. 5) or 11 mas (Fig. 3), and $W=+0.6$ or +0.5 respectively). The final level has an amplitude of about 3 to 5 mas; beyond such values, the shape becomes more and more ellipsoidal. However, note that for $\Delta R_{\mathrm{i}}=10$ mas, the values of $W$ ranging from -0.075 up to +0.6 allows a departure from a perfect ellipsoid, with more and more marked latitudinal distortions. Figure 3 can be directly compared with the fourth graph of Fig. $5\left(\Delta R_{\mathrm{i}}=10\right.$ mas $)$, the values of $W$ ranging here from -0.6 to 0.6 instead of 0.05 to 0.6 as in the previous figure. Note, thus, that in this more realistic case high negative values of $W$ render the surface more oblate (a difference of 20 mas between the equatorial and polar radius is obtained for $W=-0.6$ ). It has been checked by 


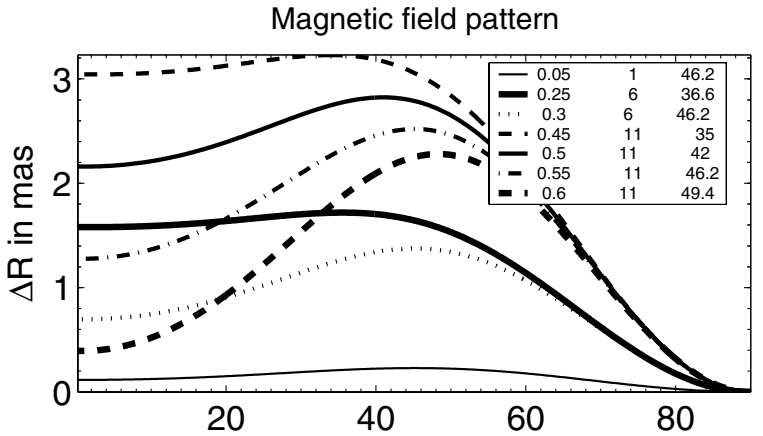

Supergranular pattern
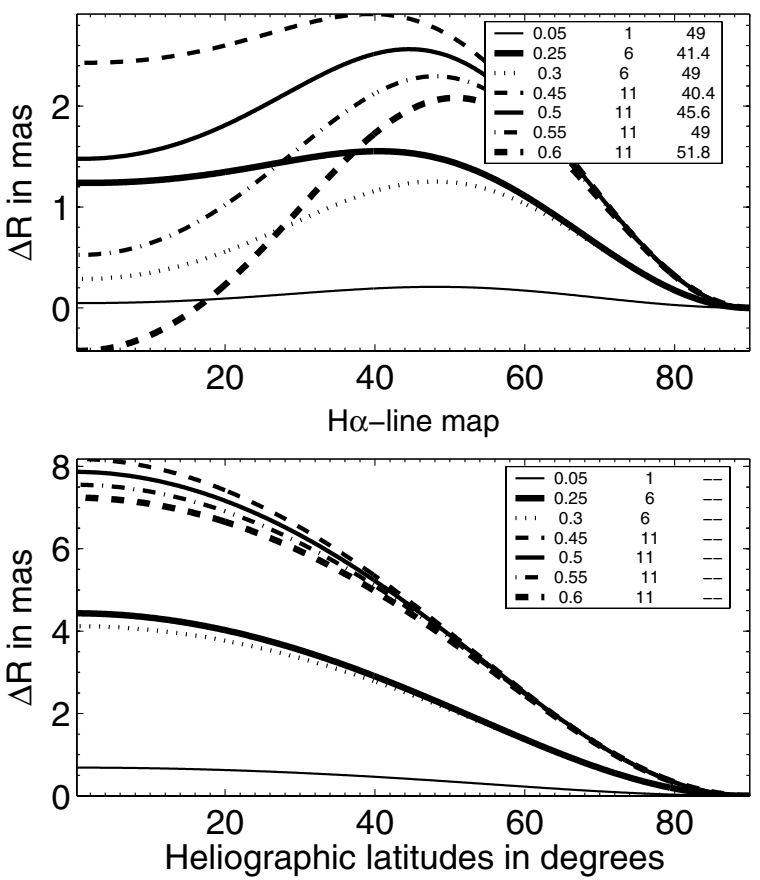

Surface plasma
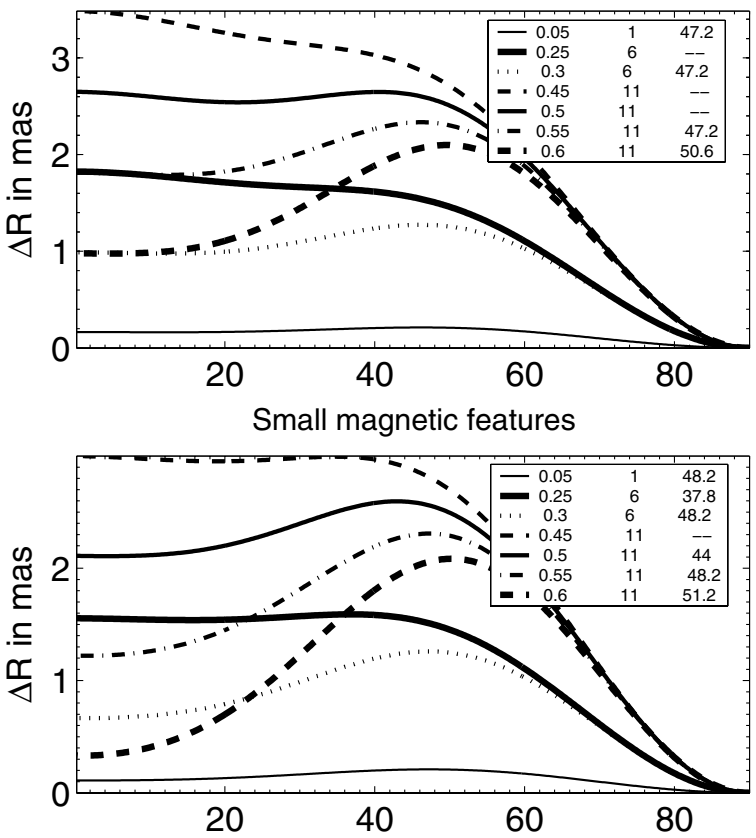

Helioseismology (Kosovichev)

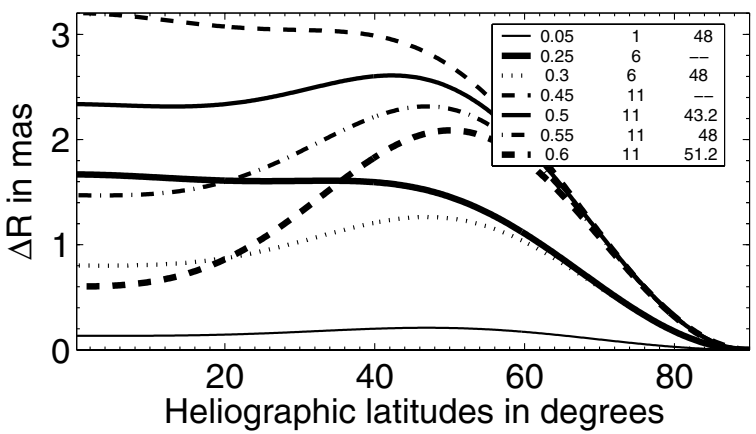

Fig. 4. Theoretical solar radius variations (by reference to the polar radius, in mas) versus heliographic latitude in degrees, for different values of the rotation rate $\Omega$ (see Table 1). The successive columns in the legend indicate the value of $W$ and of the initial $\Delta R_{\mathrm{i}}$ (in mas) and, the latitude at which the maximum of each curve occurs, repectively (a - indicates that no maximum is obtained). The successive triplets $\left(W, \Delta R_{\mathrm{i}}, \phi_{\max }\right)$ have been chosen identical to these of Fig. 3. Note the similar behaviour of the curves except for the graph labelled "H $\alpha$-line map", due to the highest altitude at which this line is formed.

plotting the maximum of the heliographic curves $\left(\theta_{\max }\right)$ versus $\Delta R_{\mathrm{i}}$ and parametrized by $W$ (such curves can be deduced for instance from the caption boxes of each plot of Fig. 5) that small values of $\Delta R_{\mathrm{i}}$ are associated with small values of $W$. Larger values of $\Delta R_{\mathrm{i}}$ yield a maximum of $\theta_{\max }$ from values of $W$ ranging between 0.3 and 0.6 . This emphasizes the need to properly measure the "asphericity-luminosity parameter $W$ " in order to discriminate between a more oblate shape (occurring at large positive values $W$ ), knowing that prolate envelopes (large negative values of $W$ ) have to be rejected. If such asphericities happen in the real Sun, it seems likely that some signature of them would be detectable.

To sum up, a pole-equator temperature difference yields a thermal-wind effect that may explain the latitudinal variation of the solar radius. To our knowledge, if a temperature gradient has been already well measured (Kuhn 1985, 1988), such a result has never been studied yet for further effects on the outer shape.

\section{Conclusions and perspectives}

To check whether latitudinal radius variations exist, a first order theory was developed, based upon the thermal wind flowing from the pole to the equator.

The main results obtained from this new theory can be summarized as follows:

- 1. The pole-to-equator temperature variations may explain observations made from the ground by different techniques and showing small solar asphericities.

-2 . The shape of the free surface depends on the initial values of the oblateness of the reference ellipsoid, and on the asphericity-luminosity parameter $W$. Their values are not independent from each other, as some values of this pair may lead to a prolate Sun. However, their values are upper and lower bounded, by 7 and 22 mas for $\Delta R_{\mathrm{i}}$, and by -0.075 and +0.6 for $W$. 

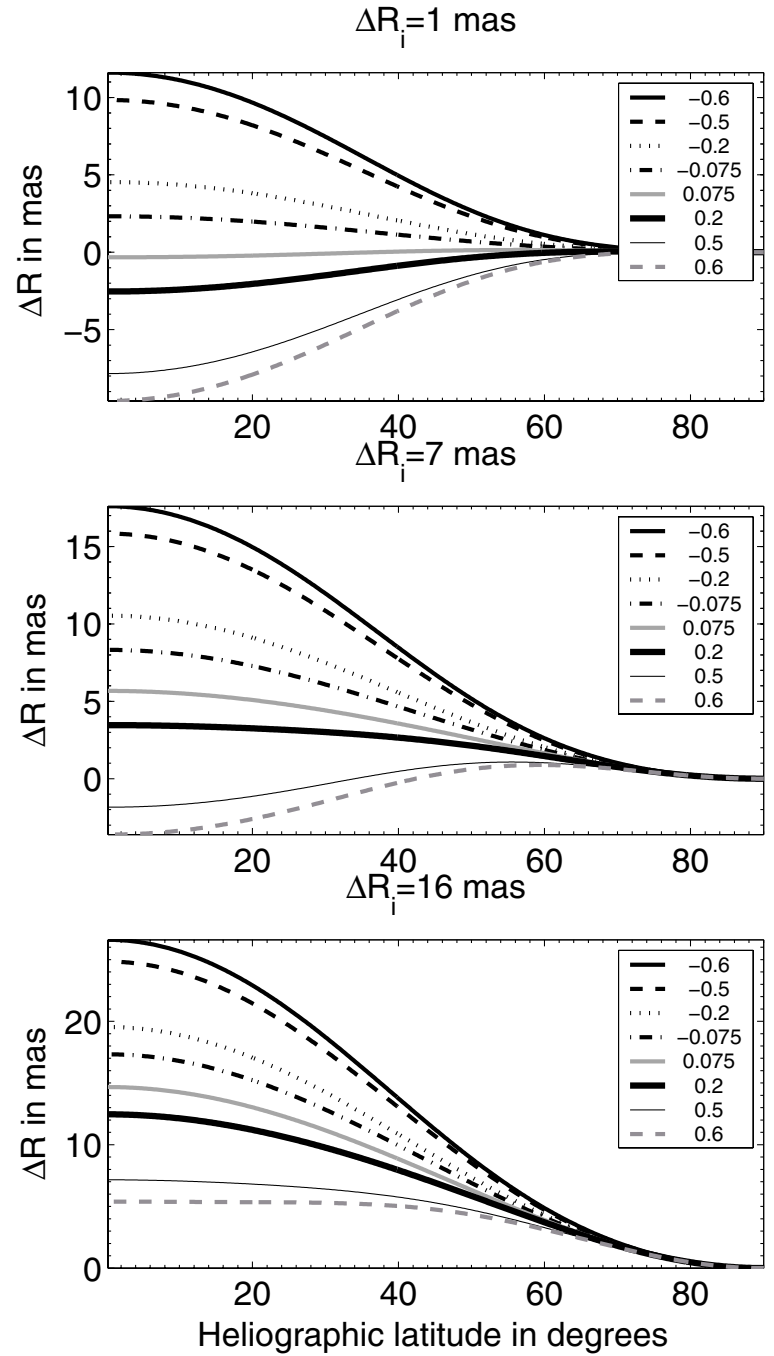
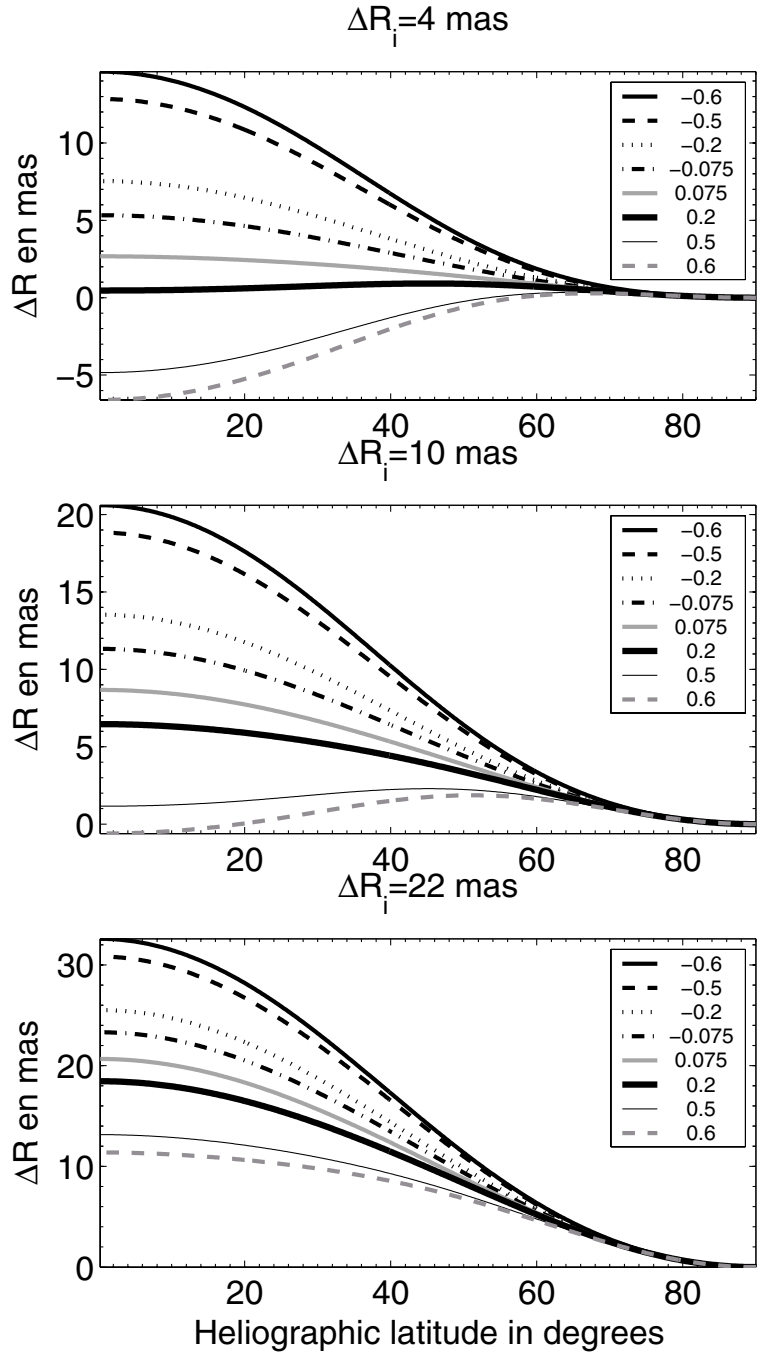

Fig. 5. Computations made with different values quoted in the literature, of the "asphericity-luminosity" coefficient $W$, ranging from -0.6 to +0.6 and for different initial values of $\Delta R_{\mathrm{i}}$. The legend in the box indicates these different values of $W$.

-3. The bulge over the royal zone is the most marked for the pair $\left[\Delta R_{\mathrm{i}}\right.$ (in mas); $\left.W\right]=[11 ;+0.5]$.

-4 . For a given value of $\Delta R_{\mathrm{i}}$, this bulge is less and less marked as $W$ decreases from +0.6 down to -0.075 .

-5 . The shape of the free surface is slightly sensitive to the values of the rotation rate, remaining globally the same, in a very thin surface layer. This merely reflects the fact that the markers used are the signatures of physical effects more or less anchored in depth.

Obviously several other effects have not been taken into account that could cancel out some of these results in a real Sun. In particular, for further calculations, it will be interesting:

- to consider the problem in two dimensions, that is to say to depict the photosphere with a certain thickness, bearing in mind that the differential rotation laws are both $r$ and $\theta$ dependent. Presently, the velocity rotation rates deduced from helioseismology are not yet accurate enough just below the surface (Di Mauro 2003) to conduct precise computations;

- to take into account the magnetic field and its pole-toequator migrations (this could also be applied to the theory of figures as described by Rozelot \& Lefebvre 2003). Such advances are currently under way.

To go further, measurements from space with sufficient resolution are essential. The measurement of the asphericities and the determination of the true helioid is one of the main goals of the future microsatellite PICARD mission (see http://smsc.cnes.fr/PICARD/Fr/, 2003), scheduled to be launched by 2008. To be more specific, PICARD will give the first simultaneous measurements of the solar diameter (in four different wavelengths), the differential rotation, the irradiance, the UV-flux and the low-degree helioseismologic modes. PICARD will thus contribute to a better knowledge of the solar machine, from the core to the surface. One of the products of these observations will be the first space measurement of the $W$ ratio and its temporal evolution. Its precise value is important to better constrain the solar models, as previously seen, and at whatever depth considered. Finally, this parameter is one of the keys for a better understanding of the solar forcing of the Earth's climate. That is also one of the reasons for which, while awaiting the launch of PICARD, we encourage the scientific 
community to support experiments in that direction, such as the SDS flights.

Acknowledgements. We thank the CNRS, the Provence-Alpes-Côte d'Azur Region and the ACRI company which support this work, and R. Feldman for her assistance with the English language. We also warmly thank the referee for pertinent comments and constructive suggestions.

\section{References}

Allen, C. W. 2000, in Astrophysical quantities, fourth edition (Springer press)

Andrei, A. 2002, personnal communication

Basu, S., Antia, H. M., \& Tripathy, S. C. 1999, ApJ, 512, 458

Beardsley, B. J., \& Hill, H. A. 1987, BAAS, 19, 1120

Corbard, T., \& Thompson, M. J. 2002, Sol. Phys., 205, 211

Dearborn, D. S. P., \& Blake, J. B. 1980, ApJ, 237, 616

Deng, Y., Wang, J., \& Harvey, J. 1999, Sol. Phys., 186, 13

Dikpati, M., \& Gilman, P. A. 2001, ApJ, 551, 536

Dikpati, M., \& Gilman, P. A. 2002, ApJ, 576, 1031

Di Mauro, M. P. 2003, in The Sun's surface and subsurface (Springer press), 599, 31

Duvall, Jr. T. L. 1980, Sol. Phys., 66, 213

Emilio, M., Kuhn, J. R., Bush, R. I., \& Scherrer, P. 2000, ApJ, 543, 1007

Floyd, L. 2003, in The Sun's surface and subsurface (Springer press), 599, 109

Godier, S., \& Rozelot, J. P. 2001, Sol. Phys., 199, 217

Gölbaşi, O., Chollet, F., Kiliç, H., et al. 2001, A\&A, 368, 1077

Gough, D. O. 2001, Nature, 410, 313

Gough, D. O., \& McIntyre, M. E. 1998, Nature, 394, 755

Irbah, A., Bouzid, A. E., Lakhal, L., et al. 2003, in The Sun's surface and subsurface (Springer press), 599, 159

Jilinski, E. G., Puliaev, S., Penna, J. L., Andrei, A., \& Laclare, F. 1999, A\&AS, 135, 227

Komm, R. W., Howard, R. F., \& Harvey, J. W. 1993, Sol. Phys., 145, 1 Kosovichev, A. G. 1996, in Sounding solar and stellar interior, IAU, 97

Kuhn, J., Libbrecht, K. G., \& Dicke, R. H. 1985, ApJ, 290, 758

Kuhn, J. R. 1988, ApJ, 331, L131

Kuiper, J. 1972, in The Sun (Chicago University press)
Laclare, F., Delmas, C., Coin, J. P., \& Irbah, A. 1996, Sol. Phys., 166, 211

Lefebvre, S., \& Rozelot, J. P. 2003, contributed paper at COSPAR General Assembly Houston, 10-13 October 2002

Lydon, T. J., \& Sofia, S. 1996, Phys. Rev. Lett., 76, 177

Makarov, V. I., Tlatov, A. G., \& Callebaut, D. K. 1997, Sol. Phys., 170,373

Noël, F. 1999, A\&A, 343, 1001

Noël, F. 2002, A\&A, 393, 667

Noël, F. 2003a, in The Sun's surface and subsurface (Springer press), 599,181

Noël, F. 2003b, A\&A, in press

Pap, J. M., Rozelot, J. P., Godier, S., \& Varadi, F. 2001, A\&A, 372, 1005

PICARD website 2003, http://smsc. cnes.fr/PICARD/Fr/

Reis Neto, E., Andrei, A. H., Penna, J. L., Jilinski, E. G., \& Puliaev, S. P. 2003, Sol. Phys., 212, 7

Ribes, E., Bearsley, B., Brown, T. M., et al. 1991, in The Sun in Time, ed. C. P. Sonnett, M. S. Giampapa, \& M. S. Matthews (Tucson: AZ press), 59

Rösch, J., Rozelot, J. P., Deslandes, H., \& Desnoux, V. 1996, Sol. Phys., 165, 1

Rozelot, J. P. 2001, JASTP, 63, 375

Rozelot, J. P., \& Bois, E. 1998, ASP Conf. Ser., 140, 75

Rozelot, J. P., \& Lefebvre, S. 2003, in The Sun's surface and subsurface (Springer press), 599, 4

Rozelot, J. P., Lefebvre, S., \& Desnoux, V. 2003a, Sol. Phys., 317, 39

Rozelot, J. P., Lefebvre, S., \& Desnoux, V. 2003b, ASP Conf. Ser., ed. A. A. Pevtsov, \& H. Uitenbroek, 286, 85

Schou, J., Antia, H. M., Basu, S., et al. 1998, ApJ, 505, 390

Snodgrass, H. B. 1983, ApJ, 270, 288

Snodgrass, H. B., Howard, R., \& Webster, L. 1984, Sol. Phys., 90, 199

Snodgrass, H. B., \& Ulrich, R. 1990, ApJ, 351, 309

Sofia, S., O’ Keefe, J., Lesh, J., \& Endal, A. 1979, Science, 204, 1306

Sofia, S., \& Endal, A. 1980, in The ancient Sun, ed. R. Pepin, J. Eddy, \& R. B. Merrill (Pergamon press), 139

Sofia, S., \& Li, L. H. 2001, J. Geophys. Res., 106, 12969

Spruit, H. C. 1991, in The Sun in Time, ed. C. P. Sonnett, M. S. Giampapa, \& M. S. Matthews (AZ press), 118

Tassoul, J. L. 2000, in Stellar Rotation, Cambridge Astrophys. Ser., 36

Wilson, P. R., Burtonclay, D., \& Li, Y. 1996, ApJ, 470, 621

Wittmann, A. D., \& Bianda, M. 2001, in The Solar Cycle and Terrestrial Climate, ed. A. Wilson, ESA SP-463, 113 